\title{
Qualitative Aspects of Mother- and Father-Infant Attachments*
}

\author{
MICHAEL E. LAMB \\ University of Michigan
}

\begin{abstract}
Thirty-two infants were observed twice in the Ainsworth Strange Situation procedure, once with their mothers and once with their fathers. Following the emphasis and procedures described by Ainsworth, the analysis focused on the nature of the infants' responses to separation and reunion. Raters then assessed the "security" of the infants" attachments to their mothers and fathers. Sixteen of the infants were "securely attached" to both parents, and seven had insecure relationships to both. Knowledge of the security of either parent-child relationship facilitated prediction $(p<.06)$ of the nature of the other relationship, but there was sufficient variability to indicate that a major determinant of the quality of either relationship might be the earlier interaction between that individual and the infant, rather than one prototypic relationship.
\end{abstract}

After a lengthy period of near-exclusive focus on mother-infant relations, researchers have recently turned their attention to the study of father-infant relationships as well (cf. Lamb, in press). For the most part, researchers have aimed to determine whether infants become attached to their fathers at all, whether father-infant attachments are formed at the same time as or after mother-infant relationships, and whether infants demonstrate preferences for one parent over the other (see review by Lamb, in press). The present study was concerned with a somewhat different issue: it was designed to elucidate differences among infants in the security of mother-infant and father-infant attachment, and to indicate whether infants who have secure relationships with one parent are more or less likely to be securely attached to the other parent. Security of attachment was determined via a procedure modeled after that of Ainsworth, Blehar, Waters, and Wall (in press).

Previous studies have demonstrated that most infants feared in traditional families form attachments to both their parents at around the same time (Lamb, 1976b, 1977a, 1977b), although in the period around the end of the first and the beginning of the second year, most infants show preferences for

\footnotetext{
*This research was supported by a faculty grant from the Graduate School Research Committee of the University of Wisconsin-Madison. Requests for reprints should be addressed to Michael E. Lamb, Department of Psychology, University of Michigan, Ann Arbor, Michigan 48104.
} 
their mothers, especially when they are distressed (Cohen \& Campos, 1974; Lamb, 1976a, 1976c). When they have access to only one parent, however, distressed infants appear to seek proximity to, and comfort from, either parent to the same extent (Lamb, 1976a, 1976c). While these findings indicate that infants can use either parent as a source of security, the measures used in the studies concerned were not appropriate to reveal whether there were qualitative differences in the manner in which the infants used their mothers and fathers as secure bases.

Toward the end of her longitudinal investigation of infant-mother attachment, Ainsworth developed a laboratory procedure known as the Strange Situation. This procedure was designed to elucidate individual differences in the quality or security of infant-mother relationships by subjecting infants to increasing stress occasioned by two separations from their mothers, and by the entrance of a strange adult. The focus was on the infants' responses to separation from, and, more importantly, reunion with, their mothers. Research conducted by Ainsworth and her colleagues (see review by Ainsworth et al., in press) revealed that infants' behavior in the situation can be predicted from information concerning maternal sensitivity early in the infants' lives (Blehar, Lieberman \& Ainsworth, 1977), that there are consistent relations between the behavior of infants at home and in the Strange Situation (Ainsworth, Bell, \& Stayton, 1971, 1972), and that there is remarkable longitudinal consistency in the manner in which infants behave in the Strange Situation (Waters, 1977).

Though the Strange Situation procedure has been employed in research on father-infant attachment (Feldman \& Ingham, 1975; Willemsen, Flaherty, Heaton, \& Ritchey, 1974), no attempts have been made to focus on the qualitative aspects discussed by Ainsworth and her colleagues, nor to compare the father-infant and mother-infant relationships of individual infants. This was the goal of the present study. Concern with this issue arose, not only from a desire to learn whether the qualitative differences that characterized mother-infant relationships were also evident in father-infant relationships, but also from a wish to determine whether the nature of the infant's relationships with its two parents were similar to one another. Similarities between the mother-infant and father-infant attachments of individual infants might be expected, if one shared Freud's belief that the mother-infant relation served as the "prototype of all later love relationships" (Freud, 1949, p. 40), since qualitative aspects of parent-child relations (such as security of attachment) appear to come closest to what Freud had in mind when he spoke of relational "prototypes." Evidently, complete evaluation of Freud's hypothesis would require a longitudinal investigation: the present study sought merely to evaluate the worth and appropriateness of extensive research of this nature in the future. 
In sum, the research was designed to see whether Ainsworth's Strange Situation procedure could be used to explore individual qualitative differences in the nature of father-infant as well as mother-infant attachments, and to determine whether there were significant similarities between the quality of the relationships enjoyed by young infants with their mothers and fathers.

\section{METHOD}

\section{Subjects}

Sixteen male and 16 female one-year-olds and their parents served as participants in this study. Subjects were recruited from published birth records by means of a contact letter, followed by a telephone call. Seventy percent of the families contacted agreed to participate, a rate higher than that reported by others using the same records in studies of social and perceptual development. The sample was composed mainly of infants from middle-class homes, reflecting the characteristics of the university town in which the research was conducted. On the 7-point Hollingshead (1957) Occupational Scale, $50 \%$ of the families were in Class I, $25 \%$ in Class II, $16 \%$ in Class III, and $3 \%$ ( 1 family) in each of Classes, IV, V, and VI. Sixty-seven percent were first-borns, $21 \%$ were second-borns, and $12 \%$ were later-borns.

\section{Procedure}

The procedure followed (see Table 1) was identical to that described by Ainsworth and her colleagues (e.g., Ainsworth et al., in press), except that (1) infants were observed twice-once with their mothers and once with their fathers, and that (2) Episode 7, which lasted for $3 \mathrm{~min}$ in Ainsworth's initial study, lasted for 2 min in the present study. The procedure involved six 3-min episodes and a final 2-min episode. The behavior of the infants was monitored as they were subjected to gradually increasing stress occasioned by two brief separations from the parents, and two entrances by a strange adult, with whom the infant was left alone. Parents were told that we were interested in seeing how infants interacted with their mothers and fathers. They were unaware that our focus was on the infants' responses to separation and reunion. The parent seen first with the infant was not told to withhold any information from the other. Such a strategy appeared unnecessary, since the parents were both instructed to be responsive but not initiatory, and since the focus was on infant behaviors that are not readily manipulable.

With few exceptions, all infants were observed twice at the same time of day. The two sessions were one week apart, and the identity of the parent seen 
TABLE 1

Procedure

\begin{tabular}{cll}
\hline Episode" & Persons present & Entrances and exits \\
\hline 1 & Parent, baby & Stranger enters \\
2 & Parent, baby, stranger & Parent leaves \\
3 & Baby, stranger & $\begin{array}{c}\text { Parent reenters; } \\
\text { stranger leaves }\end{array}$ \\
4 & Parent, baby & Parent leaves \\
5 & Baby & Stranger reenters \\
6 & Baby, stranger & Parent reenters; \\
stranger leaves
\end{tabular}

"Episodes 1 to 6 were of 3 min duration. However, as in the previous studies, Episodes 3,5, and 6 were abbreviated if the babies were inconsolably distressed, while Episode 4 was extended where necessary to ensure that the infants were reengaged in exploration of the toys before the parent departed. Episode 7 lasted $2 \mathrm{~min}$.

first was varied systematically: Half the infants were seen with their mothers first, half with their fathers. Two strangers were involved; the order in which the strangers were seen, and the identity of the parent with whom they were paired was varied systematically. There were thus 4 boys and 4 girls in each of the four Order of parent $\times$ Order of stranger cells.

The sequence of episodes within the Strange Situation procedure was not varied, since the particular sequence was selected by Ainsworth and Wittig (1969) in order to subject the infants to gradually increasing stress. They sought this because of their belief that it is in the reunions following stressful separations that individual differences in the quality of infant-parent attachment become apparent. The standard sequence has been employed in all research involving the Strange Situation (see review by Ainsworth et al., in press).

After the parent reviewed the procedure, $\mathrm{s} /$ he entered a $6.0 \mathrm{~m} \times 7.5 \mathrm{~m}$ observation room, and set the child on a designated spot in the center of an array of 26 toys. The parent then sat in a designated chair $2.5 \mathrm{~m}$ from the child. $\mathrm{S} /$ he was instructed to be responsive to the child's initiatives but not to initiate interaction, unless the child was distressed and $s /$ he wanted to reengage its interest in the toys. In cases where the infants sought to be held, the parents were instructed to pick up and comfort the babies and then attempt to rekindle the infants' interest in the toys, before returning to their chairs. 
From behind the one-way window which extended along the entire length of one wall, a videotaped record of the baby's behavior was made. The sound track was recorded via microphones mounted in the ceiling. The door through which the parent entered and exited, and the chairs in which the parent and stranger sat were all along this wall, thus assuring the camera the best possible view of the infant's face when in interaction with the adults.

The strangers were two female undergraduates. When the stranger entered in Episode 2, she sat in a chair $2.5 \mathrm{~m}$ from the parent and $2.5 \mathrm{~m}$ from the child's initial starting point. In Episode 2, she was at first quiet ( $1 \mathrm{~min}$ ), then chatted to the parent $(1 \mathrm{~min})$, and then engaged the infant in play until the parent left the room unobtrusively. In Episodes 3 and 6 she attempted to soothe the infant when necessary; when unnecessary, she withdrew to her chair and remained responsive, but not initiatory.

The videotapes were subsequently. reviewed by two trained coders. Although we attempted to apply the category definitions described by Ainsworth et al. (in press), their scoring procedure was replaced by a coding scheme in which the presence or absence of certain criterial behaviors in the separation and reunion behaviors was noted. The three separation episodes $(3,5,6)$ were separately searched for the occurrence/nonoccurrence of protest (separation distress), and two types of active search behavior: going to the door and going to the parent's chair. Also noted was the Stranger's ability to soothe the child, and whether or not the episode was curtailed.

In each of the two reunion episodes, meanwhile, the coders noted whether the infant approached the parent, and whether this was partial (baby did not come within a $3 \mathrm{ft}$ radius of the parent), or delayed more than $15 \mathrm{sec}$; whether the infant touched the parent; indicated a wish to be held; greeted the parent with a smile or cry; whether it was held by the adult; and if so, whether it resisted being put down. The coders also judged whether or not the child gave evidence in each of these episodes of proximity or interaction avoidance; and interaction or close-contact resistance. After Ainsworth et al., avoidance involved deliberate efforts by the infant to move away from, turn its back toward, or avert its gaze from the parent. Resistance involved angry attempts to push away from the parent (contact resistance), or vigorous rejection of proferred toys by pushing them away (interaction resistance).

Finally, on the basis of this coding, the coders assigned the baby to one of the three categories described by Ainsworth et al. (1971). Briefly, the A group is composed of those who are avoidant of the parent; the B group consists of infants who behave adaptively in the situation (protesting separation, and seeking proximity and contact on reunion); while the $C$ infants behave maladaptively (greeting the parent angrily and ambivalently). In essence, the occurrence of avoidance (see above) at reunion caused the infant to be placed in the A category, while the occurrence of resistant behavior (see above) upon reunion led the infant to be placed in the $C$ category. In the absence of either avoidant or resistant behavior, the infant was deemed to fall in the B category. 
In classifying the babies' behavior, the coders used detailed coding instructions supplied by Ainsworth. The coders employed the subcategories described by Ainsworth and her colleagues, although there were two few infants in the study to permit consideration of these finer categorizations in the analyses. Their use, however, facilitated classification of the infants.

Since the coding categories and classification criteria have been defined in detail by Ainsworth and her colleagues (e.g., Ainsworth et al., 1971; Ainsworth et al., in press; Blehar, 1974), and since their definitions (but not their scoring procedure) were used in the present research, the reader is referred to the aforementioned publications for further details.

\section{Reliability}

Each tape was scored by one of two trained coders. Twenty of the tapes $(31 \%)$ were independently recoded by the other coder, in such circumstances that at least one was not aware that reliability was being assessed. Reliability in the classification and in the coding of the behaviors was computed by converting into a percentage the ratio of the total number of instances on which there was exact agreement to the total number of judgments. This appears to be the most appropriate way to express coding accuracy when presence/absence judgments are involved. Intercoder agreement in the scoring of all behaviors and interaction patterns was maintained above $90 \%$ throughout the period of data analysis.

\section{RESULTS}

Chi-square and Fisher-exact tests were performed in order to assess the hypothesis that the mother-infant and father-infant relationships were qualitatively similar. Since many of the cell frequencies were less than 5 , the Fisher-exact test is more appropriate, and consequently the significance levels reported below were derived in this manner (Hollander \& Wolf, 1973). Tests were performed using data from each of the separation and reunion behavior measures, as well as the global classifications. .

Analyses indicated that infants who were securely attached (i.e., the B group) to one parent tended to be securely attached to the other, while infants who were insecurely attached (A or C) to either parent, tended to be insecurely attached to the other $(p=.055)$. The pattern of similarity is evident in Table 2. It is evident that the significant similarity is accounted for primarily by the large number of infants who were securely attached to both parents. There is considerably less similarity evident when either relationship was found to be insecure. The significant association was not evident, furthermore, when the two classes of insecure relationship (avoidant, 
TABLE 2

Classification of Maternal and Paternal Attachments $(N=32)$

\begin{tabular}{lcc}
\hline & \multicolumn{2}{c}{ Maternal attachment } \\
& Secure (B) & Insecure (A, C) \\
\hline Paternal & & \\
attachment & & \\
$\quad$ Secure (B) & 16 & 4 \\
Insecure (A, C) & 5 & 7 \\
\hline
\end{tabular}

rejecting) were differentiated, rather than being considered together as examples of insecure attachment.

Comparisons on the individual measures revealed that there were many more similarities in the infants' responses to reunion in Episode 4 than there were in Episode 7. In Episode 4, there were significant similarities in the infants' responses to maternal and paternal reunions on the following measures: requests to be held $(p<.01)$, greeting with a cry $(p<.01)$, resistance to being put down $(p<.10)$, seeking contact $(p<.10)$, avoidance of interaction $(p<.01)$, resistance of interaction $(p<.001)$, and resistance of physical contact $(p<.001)$. Since the two last-named behaviors were extremely rare, with fewer than $10 \%$ of the infants being resistant with either parent, the similarity on these measures is probably not psychologically meaningful. Not surprisingly, there was also a significant similarity in parental behavior reflected in whether or not the parents picked up the baby $(p<.05)$.

In Episode 7, by contrast, there were significant or near-significant similarities only on the variables seeking contact $(p<.10)$, and resistance to contact $(p<.001)$.

In all three of the separation episodes, the only variable to show consistent similarity was distress to such an extent that the episode had to be curtailed ( $p$ $<.01, p<.10, p<.05$ in Episodes 3, 5, and 6, respectively). With the three separation episodes considered together, there was also near-significant similarity in the occurrence of active search $(p<.07)$.

Table 3 presents the proportionate frequencies of each of the reunion behaviors, and Table 4 the proportionate frequency of the separation behaviors in each of the relevant episodes. None of the reunion or separation responses was significantly more common in relation to either parent.

Order effects. A comparison of behavior in the first and second observations was conducted using the appropriate nonparametric statistical tests. These tests revealed no significant or near significant tendency for any classification or specific behavior to be more or less common in the first or second observation. In other words, no systematic effects were attributable to 
TABLE 3

Percentage of Infants Displaying the Reunion Responses Indicated ${ }^{a}$

\begin{tabular}{lcccc}
\hline & \multicolumn{2}{c}{ Mother } & \multicolumn{2}{c}{ Father } \\
Response & Ep 4 & Ep 7 & Ep 4 & Ep 7 \\
\hline Full approach & 50 & 66 & 56 & 50 \\
Full or partial approach & 69 & 81 & 72 & 63 \\
Touch & 50 & 72 & 38 & 63 \\
Ask to be held & 25 & 50 & 28 & 34 \\
Greet with smile & 31 & 38 & 19 & 25 \\
Greet with cry & 13 & 28 & 13 & 19 \\
Held & 28 & 53 & 34 & 53 \\
Resist put down & 13 & 34 & 16 & 22 \\
Seek proximity & 59 & 81 & 69 & 59 \\
Seek contact & 31 & 53 & 34 & 44 \\
Avoid interaction & 16 & 19 & 13 & 22 \\
Avoid proximity & 6 & 6 & 6 & 6 \\
Resist interaction & 0 & 3 & 3 & 9 \\
Resist contact & 0 & 3 & 0 & 0 \\
\hline
\end{tabular}

Antries represent percentage of sample $(N=32)$ in order to facilitate comprehension.

TABLE 4

Percentage of Infants Displaying the Separation Responses Indicated ${ }^{2}$

\begin{tabular}{|c|c|c|c|c|c|c|}
\hline \multirow[b]{2}{*}{ Response } & \multicolumn{3}{|c|}{ Mother } & \multicolumn{3}{|c|}{ Father } \\
\hline & Ep 3 & Ep 5 & Ep 6 & Ep 3 & Ep 5 & Ep 6 \\
\hline Protest & 31 & 81 & 69 & 50 & 75 & 63 \\
\hline Soothe by $S$ & 16 & - & 47 & 19 & - & 44 \\
\hline Episode curtailed & 9 & 56 & 22 & 22 & 63 & 28 \\
\hline Active search & 25 & 63 & 31 & 25 & 75 & 22 \\
\hline
\end{tabular}

'Entries represent percentage of sample $(N=32)$.

the order in which the mother-infant and father-infant relationships were assessed.

\section{DISCUSSION}

It is clear from these findings that the Strange Situation procedure can be used to elucidate qualitative aspects of both mother-infant and father-infant attachments. All the coding categories, inspired as they were by those described by Ainsworth et al. (in press), proved suitable for describing fatheras well as mother-infant relationships. Roughly the same proportion of the 
relationships with each parent were described as secure (B) as were so described in earlier studies focused exclusively on mother-infant attachment.

At the level of overall classification, and in their behavior in the first reunion episode, there were several significant similarities between the behavior of the infants with respect to their two parents. In the overall classification of security of attachment, results indicated that most infants who were securely attached to one parent appeared to be securely attached to the other $(16 / 25)$. There was considerably less predictability about those for whom one relationship was rated as ambivalent or avoidant (i.e., insecure). Only 7 of the 16 infants who were insecurely attached to one parent were insecurely attached to the other as well. Furthermore, infants who were insecurely attached to both parents were no more likely to behave similarly (i.e., ambivalently or avoidantly) toward both parents than the reverse. Thus, there was no evidence that the infants had developed generalized strategies of being avoidant or ambivalent in their interpersonal relationships.

The specific behavioral measures revealed several significant similarities in the behavior of infants in interaction with their mothers and fathers in the first reunion episode (4), but only a chance number in the second reunion (7), and in the three separation episodes $(3,5,6)$. It is not clear why there was greater consistency in Episode 4, especially since this has not been found in earlier studies. Three other studies (Ainsworth et al., in press; Connell, 1976; Waters, 1977) have observed the same infants with their mothers twice in the Strange Situation. Ainsworth et al. found that babies behaved rather differently in sessions about two weeks apart. Both Waters and Connell found remarkable consistency in observations at 12 and 18 months of age at the level of overall classifications ("categorical measures"), but Waters found no consistency at the level of specific behavioral measures. None of these researchers has reported greater inconsistency in some episodes than in others.

Empirical (Waters, 1977), as well as theoretical (Ainsworth et al., in press), considerations lead us to place greater emphasis on the findings regarding overall classifications than on those concerning specific behavioral measures. We have demonstrated that it is possible to use the Strange Situation procedure to derive classifications of the quality of father-infant attachment, and that information regarding the quality of father-infant relationships is not redundant since one third of the present sample were securely attached to one parent and insecurely attached to the other. Clearly, neither the nature of the mother-infant attachment, nor the nature of the infant's preceding social experiences, led it to adopt an embracing generalized style in its interpersonal relationships (cf. Freud, 1949). Rather it seems more likely, in the light of Ainsworth's research, that the quality of the relationships which infants form to specific individuals is determined by the nature of the interaction between the infant and the person concerned, although there have been no attempts to determine whether this is true of father-infant relationships as it is of 
mother-infant attachments. The results of the present research will, I hope, stimulate the necessary research on these aspects of parent-infant attachment.

While mothers and fathers may represent stereotypically feminine and masculine modes of interaction for their infants, and thereby contribute independently to the psychosocial development of their infants by virtue of the sex-typing they facilitate (Lamb, 1977b), it is likely that the quality of the infant's interaction with each leads it to adopt interactional styles that are subsequently incorporated into a generalized style of social orientation. Thus, for example, an infant who is securely attached to both parents is more likely to engage in, and benefit from, social interaction with intra- as well as extrafamilials, than an infant whose relationship with its mother is ambivalent and with its father avoidant. Clearly, considerable research will be necessary before the merit of this formulation can be assessed: the present research merely suggests that, if generalized and enduring social styles are developed in infancy and early childhood, they are likely to reflect input not only from the mother-infant relationship, but from the father-infant relationship as well.

\section{ACKNOWLEDGMENTS}

Hildy Feen, Cynthia Neff, and Lisa Heidelberger assisted in the collection and analysis of the data.

\section{REFERENCES}

Ainsworth, M. D., Bell, S. M., \& Stayton, D. J. Individual differences in strange situation behavior of one-year-olds. In H. R. Schaffer (Ed.), The origins of human social relations. London: Academic Press, 197I.

Ainsworth, M. D., Bell, S. M., \& Stayton, D. J. Individual differences in the development of some attachment behaviors. Merrill-Palmer Quarterly, 1972, 18, 123-143.

Ainsworth, M. D., Blehar, M. C., Waters, E. C., \& Wall, S. N. The strange situation. Hillsdale, N.J.: Lawrence Erlbaum Associates, in press.

Ainsworth, M. D., \& Wittig, B. A. Attachment and exploratory behavior of one-year-olds in a strange situation. In B. M. Foss (Ed.), Determinants of infant behavior. Vol. IV. London: Methuen, 1969.

Blehar, M. C. Anxious attachment and defensive reactions associated with day care. Child Development, 1974, 45, 683-692.

Blehar, M. C., Lieberman, A. E., \& Ainsworth, M. D. Early face-to-face interaction and its relation to later infant-mother attachment. Child Development, 1977, 48, 182-194.

Cohen, L. J., \& Campos, J. J. Father, mother, and stranger as elicitors of attachment behaviors in infancy. Developmental Psychology, 1974, 10, 146-154.

Connell, D. B. Individual differences in attachment: An investigation into stability, implications, and relationship to structure of early language development. Unpublished doctoral dissertation, Syracuse University, 1976. 
Feldman, S. S., \& Ingham. M. E. Attachment behavior: A validation study in two age groups. Child Development, 1975, 46, 319-330.

Freud, S. An outline of psychoanalysis (1938). New York: Norton, 1949.

Hollander, M., \& Wolfe, D. A. Nonparametric statistical methods. New York: wiley, 1973.

Hollingshead, A. B. The two factor index of social position. Unpublished manuscript (available from the author, Department of Sociology, Yale University, New Haven, CT 06520), 1957.

Lamb, M. E. Effects of stress and cohort on mother- and father-infant interaction. Developmental Psychology. 1976, 12, 435-443. (a)

Lamb, M. E. Interactions between eight-month-old children and their fathers and mothers. In M. E. Lamb (Ed.), The role of the father in child development. New York: Wiley, 1976. (b)

Lamb, M. E. Twelve-month-olds and their parents: Interactions in a laboratory playroom. Developmental Psychology', 1976, 12. 237-244. (c)

Lamb, M. E. Father-infant and mother-infant interaction in the first year of life. Child Development, 1977, 48, 167-181. (a)

Lamb, M. E. The development of parental preferences in the first two years of life. Sex Roles, $1977,3,495-497$.

Lamb, M. E. The father's role in the infant's social world. In J. H. Stevens \& M. Mathews (Eds.), Mother/child, father/child relationships. Washington, D. C.: National Association for the Education of Young Children, in press.

Waters, E. The stability of individual differences in infant-mother attachment. Unpublished manuscript (available from the author, Department of Psychology, University of British Columbia, Vancouver, BC), 1977.

Willemsen, E., Flaherty, D., Heaton, C., \& Ritchey, G. Attachment behavior of one-year-olds as a function of mother vs. father, sex of child, session, and toys. Genetic Psychology Monographs, 1974, 90, 305-324. 